\title{
Real options econometrics for aggregate tanker investment decisions
}

\author{
George Dikos \\ Supply Chain Optimization and Planning, \\ Lafarge Cement, P.O. Box 65582, \\ 15402 Neo Psychiko, Athens, Greece \\ E-mail: Dikosg@aget.gr
}

\begin{abstract}
Understanding the limitations of the Discounted Cash flow Methodology (DCF) has resulted in increased usage of the real option analysis for ship investment decisions under uncertainty. In this paper, our contribution is two-fold: We propose an equilibrium model for explaining aggregate investment behaviour in the new building industry for tankers and provide a framework for testing the real option markup hypothesis in our model for investment decisions in new tanker vessels. Under relevant aggregation assumptions, count data models are employed to test the robustness of the real options hypothesis and its impact on investment decisions.
\end{abstract}

Keywords: real options; count data; poisson; Hausman test; investment decisions; tanker market.

Reference to this paper should be made as follows: Dikos, G. (2008)

'Real options econometrics for aggregate tanker investment decisions', Int. J. Ocean Systems Management, Vol. 1, No. 1, pp.31-44.

Biographical notes: George Dikos holds an SM in Shipping, Trade and Finance from Cass University Business School and an SM and PhD in Ocean Systems Management from MIT. He has been an Associate Visiting Professor at the Frederick University in Cyprus and a Lecturer at the University of Patras. He has worked as Project Manager for RayCap Corporation and is currently running Supply Chain Optimization and Planning for Lafarge Cement in Greece. He has consulted regularly for the shipping industry and has published several papers in Maritime Economics and Management Science.

\section{Introduction}

Real options analysis is used for evaluating investment decisions under uncertainty and irreversibility. The latter which are highly present in the shipping industry have recently motivated the application of the real option framework for ship investment decisions (Bendall and Stent, 2005; Bendall, 2002). However, it is well recognised that under competition the real option markup hypothesis may not survive (Dixit and Pindyck, 1994). Furthermore, due to significant problems of aggregation and subjectivity in the parameters, no empirical study has examined the validity of the real option theory. Any test for the real options with aggregate investment data implies simultaneous testing of the aggregating assumptions and the real options markup hypothesis. Therefore the 
objective of the paper is two-fold: Based on the specific characteristics of the tanker industry, a model for aggregate investment decisions is proposed. The model accommodates the empirical testing of the real option hypothesis in a partial equilibrium framework of investment activity in the tanker industry. The case of the 'textbook perfectly competitive' tanker industry provides a unique opportunity for testing the validity of the real option markup hypothesis.

In the last few years, option theory has been important for economics and investment decisions. After the introduction of the 'real option' value, implicit in investment decisions by McDonald and Siegel (1984), it has been well understood that uncertainty has a key role in investment. Under uncertainty and irreversibility (Dixit and Pindyck, 1994, p.142) the Net Present Value (NPV) rule is incorrect for evaluating investment decisions; uncertainty and irreversibility drive a wedge between the critical value of the project and the direct or tangible cost of investment. A great deal of theoretical work has stressed the important role of uncertainty and irreversibility on investment behaviour. However, very few applications to specific investment models have been derived in this framework and empirical research has lagged considerably behind the theoretical contributions in this literature. This research has included empirical evidence on applications of real options with firm-level data, such as the explanation of asset prices (Quigg, 1993), or entry and exit decisions (Moel and Tufano, 2002). The above studies use firm specific data, forego issues of industry equilibrium, subjectivity in the choice of the parameters and firm heterogeneity.

Due to significant problems of aggregation the theory of optimal investment decisions applies most directly to a firm or an individual decision maker. Different firms may have different action thresholds and the implications of the theory at the industry level depend crucially on the market structure of the industry. Therefore, it has been very difficult to test the real option hypothesis with industry-level (aggregate) data. In a macroeconomic setting Pindyck and Solimano (1993) tested the impact of uncertainty (volatility) on movements in investment, and they found supportive evidence for the theory. However, serious problems of aggregation still have not made it easy to test the 'option value multiple' (Dixit and Pindyck, 1994, p.184) hypothesis, namely that the option value of waiting to invest implies an action threshold, where the expected value exceeds the tangible investment cost, in industry equilibrium.

Due to the significant problems of aggregation, as well as doubt under the conditions under which the real option markup survives under competition, the real option theory has been extensively applied in shipping, but always in the form of evaluating decisions of an independent decision maker. The first application of real options in maritime economics is in the seminal PhD thesis of Goncalves (1992) which received minimal attention by the Maritime Economics community. In the contrary, the work was cited in the influential monograph by Dixit and Pindyck (1994). Motivated by this work and the salient characteristics of bulk shipping, Dixit and Pindyck (1994) devoted more than 30 pages in applying the theory for entry and exit decisions by the tanker industry. Ten years later Dikos (2004) reapplied real option models for deriving entry and exit decisions in a partial equilibrium framework, rather than the standard agent-based framework and Tsolakis and Hopp (2004) applied them for the evaluation of shipping projects.

Motivated by the unique characteristics of the tanker industry and the recent applications of real options in shipping we propose a structural partial equilibrium framework for testing the real option hypothesis with aggregate data. We build on a 
remarkable result by Leahy (1993), regarding investment in a competitive equilibrium, and use a general equilibrium framework. We propose structural models of investment in the tanker industry, which accommodate the first direct test of the real option value multiple hypothesis in industry equilibrium at the industry level. It is important to stress out that this industry accommodates all the underlying assumptions of the theory, necessary for overcoming "the serious problems of aggregation" (Dixit and Pindyck, 1994, p.421). Finally, we bring our models to aggregate data of tanker vessel orders for each period; for this we use count data econometrics.

As discussed in the introduction, there is a price to be paid for the use of aggregate data: our empirical tests necessarily test jointly our aggregation assumptions and the real option markup hypothesis. Our choice of the tanker market is motivated by Dixit and Pindyck (1994, p.237), as well as its particular structure, which provides the necessary framework for most of our aggregation assumptions. As noted by Dixit and Pindyck

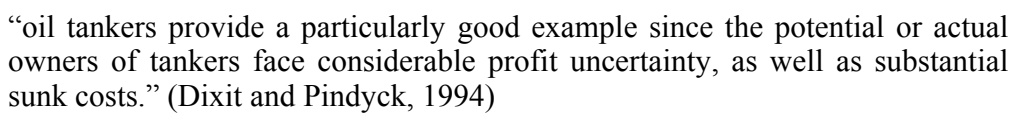

Uncertainty stems from the fact that tanker rates (the revenue per day for employing the tanker) are very volatile and fluctuate, on the one hand due to oil prices and on the other hand, due to geographical changes in the distribution and consumption of oil. Furthermore, the perfectly competitive nature of this market, as well as the existence of organised markets, provides unique necessary economic assumptions for the derivation of the model.

The paper makes a three-fold contribution: we use the underlying theory in a proper empirical context (data and methods); we use a structural approach for modelling investment behaviour in a competitive equilibrium; we employ several tests on the specification of our empirical models and assess the validity of the hypothesis of interest; we do all this in an encompassing framework, in the sense that our tests are well defined within the context of our empirical model and the empirical model itself is well defined within the context of economic theory. Furthermore, the merit of the approaches that we propose in this paper is that they maintain the use of industry level data as indicators of investment activity, and they accommodate structural estimation methods.

The rest of the paper is organised as follows. In Section 2 we present our model and put them in the context of the tanker industry; in Section 3 we describe our data set, we bring the model to the data, discuss a variety of empirical results; we offer some concluding remarks and extensions to the current research in Section 4.

\section{Model specification}

It is a remarkable result by Leahy that finds applicability in the tanker industry due to its unique salient characteristics that rescues the real option analysis under competition. However, Leahy's remarkable myopic equivalence theorem holds under very restrictive conditions as discussed in Dikos (2004) and Dixit and Pindyck (1994). Adland et al. (2006) apply Leahy's framework for deriving trading rules for generalised processes. However, a note of caution is required as Leahy's theorem holds only for the log-normal process and all firms in Leahy's equilibrium make normal profits, implying that there is no space for improvement through trading rules. 
Following Rust (1995) there are two approaches one can follow in order to model investment behaviour: The first is the 'top down' approach, where investment is computed by using a measure of a hypothetical continuous aggregate capital stock $\mathrm{K}$ and optimal investment policies arise as a result of value maximisation, subject to convex costs of adjustment for capital. A significant benefit from this approach is that it allows us to use aggregate data to test the results of the models and the validity of 'q-type' investment decisions.

At this point we should stress that optimal investment policies are derived with respect to value maximisation and we can not develop a theory of investment independently of the market structure in which the firm operates. The ability to span the under maximisation value in financial markets is also crucial for the connection between 'q-type' sufficient statistics and market value. If this is the case and the market completeness assumption is adopted, then the value maximisation objective function will be unique. This observation will turn out to be crucial even in the 'bottom up' approach that will be described right now. Since the payoff of operating a ship is fairly straightforward (it is determined by the time charter rate minus the operating costs) and an organised market exists the assumption of complete markets in this case, is a realistic assumption. Under this assumption we do not need to solve a discrete time dynamic programming problem in order to determine the value function of the optimal investment policies. This value function is determined by the assets traded in financial markets. This takes the burden of estimating the parameters of the value function.

An undoubted fact is that shipping investment decisions are discrete count decisions. As such count data methods, as introduced in the seminal paper by Hausman et al. (1984) are the natural candidate for bringing our model to the data.

\subsection{The efficient operator specification}

The shipping industry has some unique characteristics that allow us to conduct empirical tests. On the one hand, it is well known that it is one of the very few well-approximating examples of a perfect competitive industry, and on the other hand, freight rates are a sufficient statistic for the risky payoffs of operating a ship. Furthermore, the shipping industry is always suspect to unexpected regulation and pollution bills that affect directly the operating costs of vessels. Payoff uncertainty, as well as regulation and policy uncertainty imply that agents commit themselves to large-scale irreversible investments, when ordering a ship. From this point of view this industry has some unique characteristics that allow us to test the importance of irreversibility and uncertainty on the birth of investment decisions.

Perfect competition and the simple structure of the payoffs still do not resolve the issue of aggregation for firm heterogeneity. Firm heterogeneity may arise from the different running costs of different operators, preferential finance terms to credible investors and finally from the ability of the manager to achieve a long-term time charter rate for the ship, before placing the order to the shipyard. In this paper, we shall tackle aggregation following the count data econometric specification, introduced by Hausman et al. (1984). In all three models we shall specify that the total number of investment decisions follows a generalised Poisson process $P(\lambda t)$ with intensity depending on a set of factors $X_{t}$. This parametric model will be estimated using the conditional Poisson model introduced in the seminal paper by Hausman et al. (1984). 
The same model was used by Becker and Henderson (2002) with a very intuitive discussion on the microeconometric foundation of such aggregate-type models.

Following the 'bottom-up' approach to investment decisions, Becker and Henderson (2000) argue that at each point in time, there is a supply of agents willing to place orders for new vessels. They argue that this supply relation is upward sloping in positive NPV values. Since it is well established that NPV is NOT the appropriate maximising value under uncertainty and irreversibility, we shall assume that the supply of agents willing to commit themselves to shipping investment decisions is a positive function of the optimal value under uncertainty, as derived by Dixit and Pindyck (1994) and Hausman (1999). As we move up the supply curve, the higher this critical value, the more agents will place orders for new ships. Furthermore, the curve may shift outwards in periods where sources of finance are more accessible than in other periods. In the case where the underlying risk factors depend on unobservables (stochastic volatility), then the value function will depend on unobservables, too and this will shift the supply curve. The demand curve, or the number of shipyards willing to commit capacity in order to construct a ship within a prespecified period, depends on the magnitude of government subsidies and uncertainty.

Total births of investment orders are then determined by the intersection of supply and demand. This gives a reduced form equation:

$$
B_{j t}=B\left(X_{j t} ; f j+e_{j t}\right)
$$

where Bjt are the orders placed for ship type $j$ at time $t$ and $X_{j t}$ is a vector including the critical real option investment rule, the accessibility of finance sources and other macroeconomic variables and $f j$ are ship type fixed effects of unmeasured time invariant features. At this point we should note that since the underlying asset, namely the spot rate is nontradable, the critical value function shall depend on the market price of risk that will be estimated as a parameter of the model. Regarding the above specification of the model, there are two issues of concern as discussed in Becker and Henderson (2002). The first issue regarding the type of equilibrium this model does not apply in this market, due to perfect competition prevailing in this industry. The second issue regarding the nature of our data, namely discrete, with many zeros in periods of stagnancy and positive numbers makes the choice of the Poisson model a natural choice.

In order to make aggregation feasible and to impose more structure in the form of the intensity of the birth model, the following observation is made. At each point in time the most efficient operator has a known value, denoted by $V n$, if proceeding with placing an order for a new vessel that will cost him $I n$. This value $V n$ will be fully determined by the optimal investment rule that takes into account irreversibility and the option to wait. This rule is derived by Dixit and Pindyck (1994) and is used in his discussion of telecommunications industry regulations by Hausman (1999). The value of this project $V n$ will be a function of the offer $I n$ by the shipyard, the depreciation rate of the asset, the current price of the underlying risk -factor, which is the time charter rate, and the first two moments of this process, as well as the market price of risk.

Given this unobserved value the probability that the most efficient operator will not undertake investment will be given (assuming an extreme type-I error distribution) by the following formula:

$$
P_{\text {eff }}=\frac{1}{1+\exp (V n)} .
$$


This probability is then equal to the event that no birth of investment decisions will be observed in this period. Since this probability corresponds to the most efficient operator, if he does not undertake investment, no other operator will be expected to do so. If we now impose the additional restriction that the probability specified above should be equal to the probability of zero investment births, implied by the Poisson specification, then we have imposed a structure to the intensity of the count model, consistent with the real option literature.

From our count data specification, the probability that no births will be observed at time $t$ is given by:

$$
P_{0} \text { births }=\exp (-\lambda t) .
$$

Equating these two equations we now obtain the following parametric form for the intensity of the birth model:

$$
\lambda_{t}=-\ln \left(\frac{1}{1+\exp (V n)}\right) \Rightarrow \lambda_{t}=-\ln \left(P_{\text {eff }}\right) .
$$

Since $P_{\text {eff }}$ is the probability of the binary logit model, it is always restricted between zero and one, and as a consequence its negative logarithm is always positive, which is a necessary restriction for the intensity of the Poisson model. Furthermore, as discussed in Becker and Henderson (2000), in order to have a stable equilibrium of supply and demand for new ships, the sign of $\partial B_{j t} / \partial X_{t}$, with $X_{t}$ all the parameters that determine the value function, should be positive, which is the case indeed, for the above specification. Thus, the larger the value implicit in investing in a new vessel, the higher the probability investors will proceed, and the larger the number of observed orders (births in our model).

The above model is simple, tractable, makes estimation identifiable and corresponds to a partial Nash equilibrium. It is similar to the 'observed heterogeneity' model introduced by Berry (1992, p.899), where the probability that $N$ agents invest is equal to the probability that $N$ agents have a positive investment rule. However, there are two significant defects of this model. As noted by Berry, on the one hand it places strong restrictions on possible combinations of entering firms and on the other hand it assumes an infinite supply of the vessels at the given price, without interaction and adjustment from the shipyards to supply and demand shocks. In our calculations this model will be denoted as Model I.

\subsection{A perfect competition model}

The tanker sector is a paradigm for perfect competition. We may therefore make the assumption that all operators are equally competitive to the eyes of the econometrician, as he observes aggregate data and the probability that each of them will invest is the same, and is fully determined by the value of the investment minus the value of the option to wait. This probability, under the assumption of type I structural errors, is given by:

$$
\pi \text { invest }=\frac{\exp (V o p t)}{1+\exp (V o p t)} .
$$


The total number of ships ordered in each period is then given by the count variable:

$$
Y=\sum_{i=1, n} B_{i}
$$

where $B_{i}$ is the outcome of each agent, and the total number of agents is $n$. If the total number of agents is given by a Poisson process, since the outcome of each agent is an identically distributed Bernoulli trial, the total number of ordered ships will follow a Poisson process, with intensity $n \ldots \pi$. This specification allows a more flexible parametrisation of the aggregate Poisson count model, since the number of operators, may depend on other variables, too.

If we model the intensity of the number of operators as

$$
n=\exp (\alpha+\beta \cdot x) \cdot(1+\exp (V o p t)) \Rightarrow n \cdot \pi=\exp (\alpha+\beta \cdot x+c \cdot V o p t)
$$

where $x$ are some exogenous variables. This model provides us a very straightforward parametrisation of the Poisson model, and it is the familiar exponential specification of the mean, which is the main common practice in most empirical studies that deal with count data. This model of perfect competition will be named Model II. The elegant form of this model breaks down if there are uninsured risks, due to market incompleteness. As far as all operators are equiprobable in entering the market and the value of the project is uniquely spanned in financial markets, the probability of action is unique and the same for each agent. Once the spanning assets are not enough, then each agent has a different probability and the Poisson aggregation argument breaks down.

\section{Data and results}

The tanker sector has always been considered as a paradigm of perfect competition. Investing in a new tanker requires a significant amount of capital, whose main source is bank finance. Shipping cycles exhibit significant variability and no individual has ever gained enough market power, in order to control freight rates.

Regarding the data, the main source is Marsoft, Boston Inc., and it is the same source used by Dixit and Pindyck (1994, Chapter 7, p.238). Marsoft provided the orderbook (the orders placed for the construction of new vessels) for tanker ships (oil product carriers). This data set is accurate and precise. The data set is in quarters from 1980 until 2002. This implies that we are given 91 observations for each type of tanker carrier. Given the five different types of ships we have 455 observations. For this time period the data on Time Charter Rates are fully available and precise, as well as the prices of new vessels. The operating costs are fairly straightforward. A drawback is that the data on operating costs contain errors from 1980 to 1991. After that period they are known exactly.

The second source is Clarksons, London Inc. The available data are from 1993 until 2002 and are monthly. They are consistent quarterly with the Marsoft data. However, there is strong evidence that on a monthly basis there are errors.

If there are enough spanning assets, then the value of investing in a new tanker is uniquely determined and the correct investment rule is: "invest only when the value of this asset exceeds the option to wait". I will name this excess value, the real option value (Vopt hereafter) and it will be derived in line with Dixit and Pindyck (1994). 
Vopt depends not only on the underlying process for the time charter freight rates (which will be the lognormal, in line with Dixit and Pindyck), but also on the presence of bubbles. If all risks are traded, then this value is unique.

Regarding the calculation of the real option value we follow closely Dixit and Pindyck (1994, Chapter 7, p.238) and their discussion on tankers. The 'dividend' rate $\delta$ is taken equal to 0.02 (since the growth of time charter rates $\delta$ is closer to 0.02 than 0 in their analysis and the risk adjusted return to shipping $\mu$ is closer to 0.04) and the depreciation rate, is taken equal to 0.03 , since ships have a life time of 30 years. There is however significant evidence that depreciation rates depend on market conditions in this industry. They use a real option markup of $m=2.5$ in their discussion, but this markup is correct only if depreciation is omitted. As pointed out in their table in page 204, the existence of depreciation lowers the markup. Therefore, for $\sigma=0.2$ and the above parameters, the correct choice for the markup тир seems to be mир $=1.30$. Finally, regarding the 'dividend' payout rate $\delta$, a value of 0.02 is mainly consistent with the dividend ratios of most listed shipping companies. Later on, we shall not impose any specific values on these parameters and instead we shall estimate the implied parameters. If the real option markup hypothesis holds, the implied parameters have to generate a mup at least higher than 1 . Then the excess real option value which appears as a regressor is:

$$
V o p t=\frac{P}{\delta+\lambda}-\operatorname{mup} I .
$$

In the above formula $I$ is the price of the new vessels and $P$ is the revenue per year obtained from the 1 year time charter rate minus operating expenses. The calculation of the above formula for ships is accurate, since the value $I$ is known and the revenue from employing the ship from 1 year is known from the 1-year employment rate. The above formula imposes a linear restriction on the revenue (that equals the time charter rate minus the operating expenses) and the price of a new vessel. The whole point in testing whether the real options markup hypothesis is valid in this data set simply falls down in testing whether the linear restriction imposed in equation (8) is valid. Therefore, what we really have to test is the robustness of this linear restriction, implied by the real option Theory. This will be our last task, after we have specified our structural model.

Before testing the first model we should note that the number of ships ordered from 1980 until 2002 is split in five different categories. Handymax, Panamax, Aframax, Suezmax and VLCC's are the five different categories, classified on the transportation capacity of each category. Now let us test the first Model.

\subsection{Model I}

Given the efficient operator identification condition

$$
\lambda t=-\ln \left(\frac{1}{1+\exp (V n)}\right) \Rightarrow-\ln \left(P_{\text {eff }}\right) \Rightarrow \lambda t=\ln (1+\exp (V o p t))
$$

the number of ships ordered in each period are

$$
Y \approx P(\lambda)
$$


or

$$
P(Y=k \mid \lambda(x))=\exp (-\lambda(x)) \cdot \frac{(\lambda(x))^{k}}{k !}
$$

with

$$
\lambda=\lambda t \cdot a \Rightarrow \lambda=\ln (1+V o p t) \cdot a \Rightarrow \lambda=\exp (\ln (\ln (1+V o p t))+a) .
$$

Since we observe quarterly data and we do not know the decision-making frequency of the most efficient operator, we have to add a constant term in the exponential specification. This model is very restrictive since it imposes a coefficient of unity for the logarithm of the probability of zero investment, which is not the case in the specification of Model II. We shall now estimate this model by doing pooled maximum likelihood estimation, in line with HHG (1984). The data on new ships ordered contain 455 observations with 65 zero counts and a maximum value of ships ordered 66 (small size 60-DWT). Although our data set contains a significant number of zero orders, the average of ordered ships is 7.55 and the associated DWT is 0.71 million tons. There are two crucial observations at this point: On the one hand, the larger the tonnage category of the ship, the less the order counts observed in each period, and on the other hand once in the two periods of high freight rates investment 'counts' appear to be high. This is the main reason why, despite the big number of zero counts (15\%) the average as observed in the descriptive statistics in Table 1.

Table 1 Descriptive statistics

\begin{tabular}{lccccc}
\hline Variable & Obs & Mean & Std. dev. & Min. & Max. \\
\hline Ships & 455 & 7.559471 & 9.296905 & 0 & 66 \\
DWT & 455 & 0.7106032 & 0.9347692 & 0 & 7.44 \\
\hline
\end{tabular}

Number of ships ordered is 7.55. Another crucial fact is that for all our observations, time charter rates are always significantly higher than operating costs. However, only in periods when they are significantly higher, investment counts are positive. These observations are indicative, that on the one hand investment in large vessels and uncertainty affect investment decisions and intuitively they provide supportive evidence for choosing the real option value as an investment statistic.

We now do pooled Poisson maximum likelihood using the negative logarithm of the "no-investment most efficient operator" probability, which we then compare with the NLLS estimates under the exponential specification and with robust standard errors. Although it is well known from the QMLE literature that maximum likelihood is still consistent, provided the conditional mean is correctly specified, a significant difference would indicate severe misspecification. The results of the estimation are displayed in Table 2.

A significant improvement of the fit of the likelihood has been gained and a Hausman type test (1978) yields 1.65. We then get the negative binomial estimates, which improve the likelihood even more. Thus, we find evidence from significant heterogeneity among operators. Finally, the predicted counts with the actual data are compared and there is clear evidence that the Poisson specification fails to capture not only the zero counts, but as well the excess counts observed for high real option values. To verify this 
observation the following diagnostic tests are conducted. Pearson's statistic normalised for the 454 degrees of freedom yields a value of 74.92313, which is supportive for the excess overdispersion of the data set.

Table 2 Model I estimation

\begin{tabular}{lcccc}
\hline Model & NLLS & PQMLE & $N B$ & $O L S$ \\
\hline Lnp & $0.3239(0.012)$ & $0.2901(0.028)$ & $0.3461(0.040)$ & $2.691(0.352)$ \\
Const & $1.553(0.027)$ & $1.622(0.067)$ & $1.520(0.071)$ & $4.468(0.380)$ \\
Log likelihood & -2413 & -1623 & -1358 & n.a. \\
Pseudo $R^{2}$ & 0.1267 & n.a. & 0.0258 & 0.1451 \\
\hline
\end{tabular}

Furthermore, the 'Goodnessof-fit' $\chi^{2}$ statistic is 3447.149 , and rejects the Poisson specification with Prob $>\chi^{2}(452)=0.0000$. Finally, the likelihood ratio for the parameter of overdisperion of the negative binomial rejects the $H 0: \alpha=0$ with probability one and $\alpha=1.080348$.

Before proceeding with testing the second model, we consider fixed and random effects models. The fixed effects model introduces a constant term for each of the five categories of ships. Intuitively, this implies that the frequency of investing orders differs among tonnage. The random effects model assumes heterogeneity among tonnage. Following Hausman et al. (1984), a Beta random effect specification is adopted, that leads to a closed-form formula for the maximum likelihood. The introduction of multiplicative effects across different categories is equal to an intercept shift, which holds only for the exponential mean specification and corresponds to different frequencies of decision making. The results are displayed in Table 3 and standard errors are reported. For a more formal derivation of the Fixed and Random Effects Specification see Cameron and Trivedi (1998, p.275).

Table 3 Model I with fixed and random effects

\begin{tabular}{lcccc}
\hline Model & NB Fixed & NB random & Poisson FE & Poisson RE \\
\hline $\operatorname{lnp}$ & $0.3802(0.0273)$ & $0.3799(0.0273)$ & $0.3859(0.0137)$ & $0.3857(0.0137)$ \\
const & $0.2142(0.1075)$ & $0.2124(0.1074)$ & n.a. & $1.473(0.3070)$ \\
Log likelihood & -1201 & -1237 & -1491 & -1528 \\
\hline
\end{tabular}

The coefficient of $\ln p$ is now slightly higher than in the previous specification and the Log-likelihood is significantly higher. All estimated coefficients are still statistically significant. The Hausman test between Poisson Random and Fixed effects does not reject the random effects specification since it yields a $\chi^{2}(1)=0.23$ and the same result is verified for the Negative Binomial Random vs. Fixed effects Hausman test with $\chi^{2}(1)=0.03$. The Likelihood Ratio test of the Negative Binomial Random Effects specification vs. the pooled estimates follows a $\chi^{2}(1)$ with value 223.30 , which indicates that the NB specification is far more suitable. Finally, by inspecting the predictions of the model it is clear that it captures successfully the low and zero counts, but it fails severely to predict the excess counts observed at the peak of the shipping cycle. Results are displayed in Table 4. Let us therefore proceed with Model II. 
Table 4 Model II

\begin{tabular}{lcccc}
\hline Model & PQMLE & NLLS & $N B(R E)$ & OLS \\
\hline ship1 & $0.0362(0.0051)$ & $0.0240(0.0057)$ & $0.0611(0.0067)$ & $0.6212(0.0806)$ \\
Vopt & $0.0135(0.0021)$ & $0.0149(00.0050)$ & $0.0130(0.0029)$ & $0.0724(0.0210)$ \\
$V^{2}$ opt & $-2.57(0.645) \mathrm{e}-05$ & $-2.66(0.100) \mathrm{e}-05$ & $-2.64(0.653) \mathrm{e}-05$ & $-1.189(0.433) \mathrm{e}-04$ \\
Newprice & $-0.0158(0.0037)$ & $-0.0291(0.0131)$ & $-0.01114(0.0027)$ & $-0.0551(0.0172)$ \\
Accident & $-0.1517(0.1483)$ & $0.0854(0.1617)$ & $-0.3551(0.1757)$ & $1.405(1.720)$ \\
Lrate & $-0.02349(0.018)$ & $0.00602(0.019)$ & $-0.0325(0.0207)$ & $0.0619(0.132)$ \\
Vopt; lag & $-0.002072(0.00118)$ & $-0.002129(0.00150)$ & $-0.0008354(0.00206)$ & $-0.0290(0.0129)$ \\
Const & $2.444(0.203)$ & $2.787(0.401)$ & $2.097(.2351)$ & $4.734(1.541)$ \\
Pseudo $R^{2}$ & 0.3623 & n.a. & 0.0980 & 0.5278 \\
Log likelihood & -1758 & -1497 & -1255 & n.a. \\
\hline
\end{tabular}

\subsection{Model II}

We now assume that the probability of a positive count is the same for all operators, which is a fairly good assumption for a competitive market like the market of crude oil carriers. As a consequence the specification of the Poisson model has the following form:

$$
P(Y=k \mid \lambda(x))=\exp (-) \cdot \frac{(\lambda(x))^{k}}{k !}
$$

with

$$
\lambda(x)=\exp (\alpha+\beta \cdot x+\gamma \text { Vopt })
$$

Regarding the exogenous variables $x$ that determine the expected number of investors, we use the following: A lag of the number of ships ordered one-quarter before, a squared value of Vopt and a dummy variable for the accident of Erika in December 1999. Since the predominant source of ship finance is the banks, we also include the lending rate in the regressors. We now run Poisson likelihood estimation (with robust standard errors), NLLS, Negative Binomial with Random Effects and OLS with robust errors.

It is clear that the real option value appears statistically significant for all the above specification. Conducting some diagnostic tests, the Poisson $\chi^{2}(n-k)$ has a value of 2140 and rejects the Poisson specification with probability one. Pearson's statistic normalised for the degrees of freedom has a value 44.3 that indicates severe overdispersion and the likelihood ratio test on the overdispersion parameter of the negative binomial rejects the $H 0: \alpha=0$ with probability one. By inspecting the predictions of the negative binomial, the model fails to predict the zero counts as well some excess counts, especially for the lighter categories, where the most 'excess events' are observed.

The results of the four different specifications are presented in Table 5 and it is clear that for all these specifications the 1-year time charter rate, the operating expenses and the price of the new vessel are statistically significant. The tcrate is always positive and the other two variables are negative as expected. What is even more impressive is that the magnitude of the coefficient of the operating expenses is of the same significance and 
slightly higher than the corresponding coefficient of the time charter rates, which is as expected, since costs incur, even when the ships does not earn revenue (dry-dock, etc.). Furthermore, the tcrate and opex are on a per day basis. Thus, if we calculate the difference of these two coefficients and multiply it by 350 days (to take into account off-hire periods) and 365 days, respectively, the number we get is of the same significance as the coefficient of the new building price, but almost three times less, exactly as predicted by the real option literature. In order to make this point clear the estimation is repeated and instead of using tcrate and opex as regressors we use the value of the project $\mathrm{Val}$, which is given by the formula $\mathrm{Val}=$ tcrate $350-$ opex $365 / \delta+\lambda$. and then the coefficient of this variable is compared with the coefficient of the newprice. If the real option literature is correct, the coefficient of newprice has to be higher than the coefficient of $\mathrm{Val}$, which is exactly the case. Indeed, the real option markup implied by the data indicates a value close to 4 , which corresponds to an implied volatility for the underlying profit flow process of 0.40 ! For the exponential mean specification the Akaike criterium indicates that the model performs better when the real option value is used as a regressor (with a markup of 1.3 for the excess option value) than using tcrate, opex and newprice as regressors. It is now clear that on the one hand the optimal combination between the variables that determine the value of the project and the option to wait is the one predicted by the real option literature and on the other hand, not much can be gained by assuming a time-varying markup specification.

Table 5 Model II specification testing

\begin{tabular}{lcccc}
\hline Model & NLLS & PQMLE & $N B(R E)$ & OLS \\
\hline Ship1 & $0.0277(0.0047)$ & $0.0393(0.0054)$ & $0.0491(0.0034)$ & $0.6196(0.0800)$ \\
Tcrate & $3.47(0.897) \mathrm{e}-05$ & $4.37(0.880) \mathrm{e}-05$ & $3.58(0.543) \mathrm{e}-05$ & $2.65(0.83) \mathrm{e}-04$ \\
Opex & $-3.83(1.87) \mathrm{e}-05$ & $-2.68(1.83) \mathrm{e}-05$ & $-2.65(1.50) \mathrm{e}-05$ & $-1.755(1.091) \mathrm{e}-04$ \\
Newprice & $-0.0293(0.0082)$ & $-0.0230(0.0046)$ & $-0.0166(0.0032)$ & $-0.1127(0.0300)$ \\
Lrate & $-0.0013(0.019)$ & $-0.0361(0.017)$ & $-0.0589(0.017)$ & $0.0252(0.1281)$ \\
Accident & $0.2157(0.2142)$ & $-0.1898(0.1682)$ & $-0.3952(0.1917)$ & $0.4585(10.700)$ \\
Const. & $2.642(0.288)$ & $2.390(0.208)$ & $1.122(0.261)$ & $4.702(1.783)$ \\
Pseudo R & n.a. & 0.3398 & n.a. & 0.5195 \\
Log likelihood & -1515 & -1820 & -1257 & n.a. \\
\hline
\end{tabular}

\section{Conclusions}

In this paper, we have demonstrated that the real option value of a project as spanned in complete markets is a sufficient statistic for characterising aggregate investment decisions. In the case of irreversibility and uncertainty the correct specification for this value is the one expected by the real options literature. The value of the project not only has to exceed the investment cost, but also the option to wait. In the case of the perfect competitive market for new tankers, the statistical results for the last 22 years have verified both these hypotheses. Furthermore, count data models (a natural specification for investment orders) have provided a very good fit to the data. A natural extension of 
this work is the application of the proposed framework for exit decisions (scrapping), as well as in other shipping industries or oil exploration projects.

\section{Acknowledgements}

I am grateful for helpful discussions with Jerry Hausman, Victor Chernozhukov, Whitney Newey, and seminar participants at the Department of Economics at MIT and Harvard University. Financial support from the A. Onassis and Eugenides Foundation is greatly acknowledged. Special thanks to Jerry Hausman for his encouragement and support during the write-up of my 14.381 Econometrics term paper.

\section{References}

Adland, R., Koekebakker, S. and Sodal, S. (2006) 'Value based trading of real assets in shipping under stochastic freight rates', Proceedings of the 10th Annual Real Options Conference, New York.

Becker, R. and Henderson, V. (2000) 'Effects of air quality regulations on polluting industries', The Journal of Political Economy, Vol. 108, pp.379-421.

Becker, R. and Henderson, V. (2000) 'Effects of air quality regulations on polluting industries', The Journal of Political Economy, Vol. 108, pp.379-421.

Bendall, H.B. (2002) 'Valuing maritime investments using real options analysis', in Grammenos, C.Th. (Ed): The Handbook of Maritime Economics and Business, LLP, London, pp.642-660.

Bendall, H.B. and Stent, A.F. (2005) 'Ship investment under uncertainty: valuing a real option on the maximum of several strategies', Maritime Economics and Logistics, Vol. 7, pp.19-35.

Berry, S. (1992) 'Estimation of a model of entry in the airline industry', Econometrics, Vol. 60, pp.889-917.

Cameron, C. and Trivedi, P. (1998) Regression Analysis of Count Data, Cambridge University Press, Econometric Society Monographs, Cambridge.

Dikos, G. (2004) Decisionmetrics: Dynamic Structural Estimation of Shipping Investment Decisions, Unpublished PhD Thesis, MIT, Cambridge, MA.

Dixit, A. and Pindyck, R. (1994) Investment Under Uncertainty, Princeton University Press, Princeton.

Goncalves, F. (1992) Optimal Investing and Chartering Decisions in Bulk Shipping, Unpublished $\mathrm{PhD}$ Thesis, MIT, Cambridge, MA.

Hausman, J. (1978) 'Specification tests in econometrics', Econometrica, Vol. 46, pp.1251-1271.

Hausman, J. (1999) 'The effect of sunk costs in telecommunications regulation', in Alleman, J. and Noam, E. (Eds.): The New Investment Theory of Real Options and its Implications for Telecommunications Economics, pp.87-92.

Hausman, J., Hall, B. and Griliches, Z. (1984) 'Econometric models for count data with an application to patents - RD relationship', Econometrica, Vol. 52, pp.909-938.

Leahy, J. (1993) 'Investment in a competitive equilibrium: the optimality of myopic behavior', Quarterly Journal of Economics, Vol. 108, pp.1105-1133.

McDonald, R. and Siegel, D. (1984) 'The value of waiting to invest', Q.J.E., Vol. 101, pp.707-728.

Moel, A. and Tufano, P. (2002) 'When are real options exercised? An empirical study of mine closings', Review of Financial Studies, Vol. 15, pp.35-64. 
Pindyck, R. and Solimano, A. (1993) 'Economic instability and aggregate investment', NBER Macroeconomics Annual, Vol. 8, pp.259-303.

Quigg, L. (1993) 'Empirical testing of real option-pricing models', Journal of Finance, Vol. 48, pp.621-640.

Rust, J. (1995) 'Optimal replacement of GMC bus engines: an empirical model of Harold Zurcher', Econometrica, Vol. 55, pp.999-1033.

Tsolakis, S.D. and Hopp, C. (2004) 'Investment applications in the shipping industry', Proceedings of the 8th Annual Real Options Conference, Paris. 